\title{
Relationship between Bi Ventricular Systolic Function and Different Degrees of Liver Impairment
}

\author{
H.M.Mohamed, S.M.Ahmed and K.M.Abou El-Enein \\ Cardiology Dept., Faculty of Medicine, Benha Univ., Benha, Egypt \\ E-Mail:KM@yahoo.com
}

\begin{abstract}
Cardiac affection during liver disease is accompanied with hazard of acute cardiac failure and very poor prognosis after liver transplantation. We hope to evaluate the connection between the severity of heart impairment and liver disease. Hundreds of patients and Hundreds of control persons had done Trans thoracic echocardiography, TDI and ST measures. We evaluate the relation between Trans thoracic echocardiographic values and MELD and Child scores. Values of systolic function (s wave, $\mathrm{p}<0.001)$ and GLS $(\mathrm{p}<0.001)$ in addiction to values of diastolic function were observantly more reduced in hepatic patients in comparison to control persons. There were no otherness between the different sets in the using scores regarding both systolic diastolic functions, the LA size interconnected to both Child $(\mathrm{p}=0.11, \mathrm{r}=0.25)$ and MELD scores $(\mathrm{p}=0.12, \mathrm{r}=0.23)$. LVEF was largely decreased in no redeemed patients in comparison to redeemed patients $(\mathrm{p}=0.11)$. there is no correlation between degree of hepatic disease and heart impairment. So, a echo study should be done in hepatic patients prior interventional and surgical steps.
\end{abstract}

Keywords: Cardiac impairment, ST technology, Systolic and diastolic function.

\section{Introduction}

Cardiac affection during liver disease was delineative in persons having alcoholic cirrhosis, without associated heart troubles, example, a anemia or regurgitating valuvular diseases having increased cardiac output and peripheral arterial dilation [1]. That sector of patients own bad sequel, particularly in persons who will have invasive interventions example of connection between portosystimc circulation (TJPS), liver intervention, and liver implant [2,3]. Newly, impairment of diastolic function is considered to be a foreteller of death in hepatic patients with liver cirrhosis [4]. Number of physicians inform that the raise of number of cases of impairment of diastolic function in the patients with end stage of liver impairment (Late cirrhosis with huge ascites), a sure relationship between the intensity of hepatic disease and the degree of heart impairment not found. Knowing this connection is not easy due to the constant alterations in the introduction of Cirrhotic cardiomyopathy. Cirrhotic cardiomyopathy was defined as 'a inveterate impairment of heart function in persons with liver disease showing by decrease contraction response to stressful conditions with or without affected the function of the diastole with electrical changes when cardiac disease is not found.

By the use of modern technique to evaluate Cirrhotic cardiomyopathy, showing distinct propagation number of cases. The theories of affection of circulation in cirrhosis are increasing progressively. At the first time, it was construes by a global peripheral vascular dilation with low peripheral vessel intensity. Following sets on hepatic patients with ascites indicated that arterial expansion happens mainly in the vessel of gastroenterology system with compensatory constriction of the blood vessels outside gastroenterology organs, including brain, kidneys, liver and spleen [6,7]. For that reasons, The physiological study is not the only suggested pathogenesis of the affection of systolic function. The pathogenesis is more complicated and many pathogenesis are included, as affection of the receptors of beta-adrenergic types, otherness of the integrity of ion channels job in cardiac cells and the injurious effect of increase production of nitric oxide $[8,10]$. The connection of those mechanisms with the advancement of hepatic impairment is hard to be evaluated.

It was hoped in this research to catch in a patients in which there is relationship between liver impairment state and the severity of cardiac deterioration evaluated with the new tools and according to the guidelines of the American Society of Echocardiography and the timing of hepatic affection evaluated by traditional scores (CHILD and MELD) $[11,12]$.

\section{Patients and methods}

This was a cross-sectional study. We studied all the diseased persons who went to the partition of gastro logy from February 2012 to November 2014. Liver impairment was known by using of notes of clinical picture, lab results, sonar and histopathological data. We not choose the circumstances which may be initially change the heart performance example: DM, HTN, chest troubles, chanciest ischemic heart disease, patient more than 70 ys and less than 25 ys, liver cancer, kidney troubles, decrease hemoglobin percent (less than $9 \mathrm{~g} / \mathrm{dl}$, thyroid dysfunction, liver affection due to alcoholic abuse, and un natural ECG findings example: abnormalities in the heart rate.

\section{Results}

Diseased persons with liver impairment presented continuously to the section of gastro logy. On the base of on the choosing information's, hundreds of Diseased persons were chosen in this research. The cause of liver disease occurred after viral infection in many of cases. A lot of cases $(78.6 \%)$ taken beta adrenergic receptors antagonists during performing trans thoracic echo. The data of performance of systole (systolic wave, p < $0.001)$ also LVGLS $(\mathrm{p}<0.001)$ ). Also the data of performance of the diastole had been affected much 
more in the diseased persons in comparison to the normal subjects. As the previous identification: the left ventricular ejection fraction less than fifty fifty percent, impairment of systole present in fourteen diseased person. By the aid of novel trans thoracic echo, systolic wave on the wall against the medial wall less than eight centimeter per second is present in thirty thirty diseased persons. The all degrees of liver impairment shows nearly the same affection of the performance in systole however any connection could be found among the left ventricular ejection fraction, GLS or myocardial performance index and CHILD items.

Impairment of the function of the diastole present sixty one percent in our research represented mainly by first degree. No connection was found between the function of the diastole and grades of liver impairment. LA size was higher in late degree of hepatic impairment. Left ventricular thickness was huge in not compensated diseased persons.

\section{Discussion}

The expansion of liver disease affecting the cardiac function varies widely between researches due to deficiency of a clear definition, changeability of the methods which needed to evaluate that sector, and the changeability of comprehensive standards.

As the recommendations of the meeting of gastro logy in Montreal To diagnose Cirrhotic cardiomyopathy there should be the following criteria: cardiac function in systole less than fifty fifty percent or decrease in the stroke volume after stress or drugs or the cardiac function in diastole showing $\mathrm{E}$ wave over $\mathrm{A}$ wave ratio less than one or dragging period more than two hundreds $\mathrm{ms}$ or IVRP more than eight milliseconds. The another helpful standards were included example increase the duration of corrected qt, unnatural chronotropic response, huge LA, over much myocardial thickness, elevated BNP, and elevated cardiac enzymes [3]. By the adoption of this opinion, the prevalence of cirrhotic cardiomyopathy in study was detected from 52 to $74 \%$ in a lot of researches $[15,17]$.

Novel methods are able to diagnose precocious signs of heart affection, chiefly affection strain function including s wave on tissue Doppler imaging and GLS on the ST technology. As the new recommendations of the trans thoracic echo also multiple researches say, The GLS is considered impaired when it more than minus eighteen also when systolic wave is lower than eight centimeter per second $[11,1]$. In our study, $16.6 \%$ of the patients had a left ventricular ejection fraction $<55 \%$, while $20.3 \%$ had a global longitudinal strain $>-18 \%$ and $43.1 \%$ with systolic wave less than eight centimeter per second. Really As we not choose except basal LGS, It was discovered $41 \%$ of the cases with inferior BLS more than minus eighteen. That result cope with the grade of decrease of systolic function as the two techniques evaluate basilar areas.

\section{Limitations of the study}

There are some limitations in this study example Absence of BNP examination As it is essential to assess Diastolic dysfunction (21). Also, exercise test whether by drugs or by exercise As it is beneficial method to shows little heart impairment.

\section{Conclusion}

Pathogenesis of heart in diseased liver persons is complicated. Also no relation among the intensity of liver impairment and degree cardiac impairment. Hence to adjust good treatment to liver diseased person, Trans thoracic echo study should be done to every diseased person prior invasive procedures also prior to hepatic donation whether liver fibrosis is present or not.

\section{References}

[1] H.J.Kowalski, W.H. Abelmann, The cardiac output at rest in Laennec's cirrhosis. J Clin Invest, Vol. 32(10), PP.1025-1033, 1933.

[2] R.N.Rabie, M.Cazzaniga, F. Salerno, The use of E/A ratio as a predictor of outcome in cirrhotic patients treated with transjugular intrahepatic porto systemic shunt. Am J Gastroenterol, Vol.104(10), PP. 24582466, 2009.

[3] M.Cazzaniga, F.Salerno, G. Pagnozzi, Diastolic dysfunction is associated with poor survival in patients with cirrhosis with transjugular intrahepatic portosystemic shunt, Vol.56(6), PP. 869-875, 2007.

[4] L.Ruiz-del-Arbol, L.Achecar, R.Serradilla, Diastolic dysfunction is a predictor of poor outcomes in patients with cirrhosis, portal hypertension and a normal creatinine. Hepatolbaltim Md, Vol.58(5), PP.1732-1741, 2013.

[5] S.Moller, JH.Henriksen, cardiovascular complications of cirrhosis, Vol.57(2), PP.268-278, 2008.

[6] M.Guevarz, C.Bru, P.Gines, Increased cerebrovascular resistance in cirrhotic patients with ascites. Hepatolbaltim Md, Vol.28(1), PP.39-44, 1998.

[7] A.Maroto, P.Gines, V.Arroyo, Brachial and femoral artery blood flow in cirrhosis: relationship to kidney dysfunction. Hepatolbaltim Md, Vol.17(5), PP.788793, 1993.

[8] N.Herringz, E.Danson, DJ.Paterson, Cholinergic control of heart rate by nitric oxide is site specific. News Physiolsciint J Physiol Prod Jointly Int Union Physiolsci Am Physiol Soc, Vol.17, PP.202-206, 2002.

[9] A.Anselmi, M.Gaudino, A.Baldi, Role of apoptosis in pressure-overload cardiomyopathy. J Cardiovasc Med Hagerstown Md, Vol.9(3), PP.227-232, 2008.

[10] Z.Szabo, M.Harangi, I.Lorincz, Effect of hyperlipidemia on QT dispersion in patients without ischemic heart disease. Can J Cardiol, Vol.21(10), PP.847-850, 2005.

[11] Rm.Lang, LP.Badano, V.Mor-Avi, Recommendations for cardiac chamber quantification by echocardiography in adults: an update from the American Society of Echocardiography and the European Association of Cardiovascular Imaging. 
Eur Heart J Cardiovasc Imaging, Vol.16(3), PP.233270, 2015.

[12] SF.Nagueh, OA.Smiseth, CP.Appleton, Recommendations for the evaluation of left ventricular diastolic function by echocardiography: an update from the American Society of Echocardiography and the European Association of Cardiovascular Imaging. Eur Heart J Cardiovasc Imaging, Vol.17, PP.1321-1360, 2016.

[13] SF.Nagueh, OA.SmisethA, CP.Appleton, Recommendations for the evaluation of left ventricular diastolic function by echocardiography: an update from the American Society of Echocardiography and the European Association of Cardiovascular Imaging. J Am Socechocardiogr off Publ Am Socechocardiogr, Vol.29 (4), PP.277-314, 2016.

[14] A.Nazar, M.Guevara, M.Sitges, LEFT ventricular function assessed by echocardiography in cirrhosis: relationship to systemic hemodynamics and renal dysfunction. J Hepatol, Vol.58(1), PP.51-57, 2013.

[15] F.Sampaio, J.Pimenta, N.Bettencourt, Systolic and diastolic dysfunction in cirrhosis: a tissue-Doppler and speckle tracking echocardiography study. Liver Intoff J Intassoc Study Liver, Vol.33(8), PP.11581165, 2013.
[16] DA,Morris, LH,Boldt, H.Eichstadt, Myocardial systolic and diastolic performance derived by 2dimensional speckle tracking echocardiography in heart failure with normal left ventricular ejection fraction. Circ Heart Fail, Vol.5(5), PP.610-620, 2012.

[17] OM.Silvestre, F.Bacal, D.De Souza Ramos, Impact of the severity of end-stage liver disease in cardiac structure and function. Ann Hepatol, Vol.12(1), PP.85-91, 2013.

[18] M.Merli, A.Calicchia, A.Ruffa, Cardiac dysfunction in cirrhosis is not associated with the severity of liver disease. Eur J Intern Med, Vol.24(2), PP.172-176, 2013.

[19] AM.Fattouh, MH.El-Shabrawi, EH.Mahmoud , Evaluation of cardiac functions of cirrhotic children using serum brain natriuretic peptide and tissue Doppler imaging. Ann Pediatrcardiol, Vol.9(1). PP. 22-28, 2016.

[20] X.Li, S.Yu, L.Li, Cirrhosis-related changes in left ventricular function and correlation with the model for end-stage liver disease score. Int J Clinexp Med, Vol.7 (12). PP. 5751-5757, 2014.

[21 A.Salari, A.Shafaghi, M.Ofoghi, Diastolic dysfunction and severity of cirrhosis in nonalcoholic cirrhotic patients. Int J Hepatol, PP.1-6, 2013. 